\title{
UMA APLICAÇÃO DE CONJUNTOS DIFUSOS NA OTIMIZAÇÃO DO PROGNÓSTICO DE CONSENSO SAZONAL DE CHUVA NO NORDESTE DO BRASIL
}

\author{
EMERSON MARIANO DA SILVA ${ }^{1,2}$, JOSÉ MARIA BRABO ALVES ${ }^{1}$, \\ MARCO AURÉLIO HOLANDA DE CASTRO ${ }^{1}$, VICENTE P.P.B. VIEIRA ${ }^{1}$ e \\ JOSÉ NILSON B. CAMPOS ${ }^{1}$
}

${ }^{1}$ Universidade Federal do Ceará, Departamento de Engenharia Hidráulica e Ambiental

Campus do Pici, Bloco 713, Fortaleza, CE, CEP: 60455-460

${ }^{2}$ E-mail: emerson_mariano@hotmail.com

Recebido Junho 2005 - Aceito Abril 2006

\begin{abstract}
RESUMO
Esse estudo apresenta a aplicação da teoria de conjuntos difusos como ferramenta para otimizar a previsão de consenso (PC) sazonal de chuva da Região Semi-Árida do Nordeste do Brasil (RSANEB) para o período de 1985-1996. Foram utilizados como variáveis de entrada parâmetros termodinâmicos sobre e nos Oceanos Atlântico e Pacífico Tropicais. Os resultados mostraram que qualitativamente, na escala interanual, o resultado determinístico dessa técnica aplicada a PC foi capaz de prever pelo menos uma das categorias da variável de saída (total de chuva de fevereiro a maio da RSANEB). Quantitativamente, os menores erros foram observados para os anos classificados na variável de saída nas categorias de Normal (N), Chuvoso (C), e Muito Chuvoso (MC), com correlações que variam de 0,8 a 0,85 , dependendo do método de desfuzificação usado. Esta técnica permite unificar em um resultado determinístico todas as informações climáticas usadas na previsão sazonal de chuva da RSANEB, possibilitando seu prognóstico em mais de uma categoria, informando a mais provável a vir a ocorrer em função do seu nível de pertinência.

Palavras-chave: conjuntos difusos, previsão, variabilidade sazonal, chuva.
\end{abstract}

\begin{abstract}
AN APPLICATION OF FUZZY SETS AT OTIMIZATION OF THE RAINFALL SEASONAL CONSENSUS FORECAST IN BRAZIL'S NORTHEAST.

This study presents the application of fuzzy sets theory as a tool for sazonal rain forecast of SemiArid Northeast Region of Brazil for the period of 1985-1996. Thermodynamic parameters in Tropical Atlântic and Pacific Oceans were used as input variables. The results have shown that qualitatively, in an annual scale, this technique was able to forecast at least one of the categories of the output variable (total February/May rain in the of Semi-Arid Northeast Region of Brazil). Quantitatively, the smallest errors have been observed for the years classified according to the output variable in the categories of Normal (N), Rainy (C), and Very Rainy (MC), with correlation coefficients ranging fron 0.8 to 0.85 , depending on the defuzzification method used. This technique allows for the unification of all the climatic information used in the rain foreceast of Semi-Arid Northeast Region of Brazil and leads to a forecast grouped in more than one category, informing the one most likely to occur as a function of its membership value.
\end{abstract}

Keywords: fuzzy sets, forecast, seasonal variability, rainfall. 


\section{INTRODUÇÃO}

Definidos conceitualmente como conjuntos difusos ou nebulosos (Fuzzy Sets), a lógica Fuzzy foi proposta por Zadeh (1965, 1978), como uma generalização do conceito da teoria clássica dos conjuntos (Galvão, 1999), um sistema lógico não clássico, com grau de pertinência dos elementos definidos no intervalo entre $[0,1]$.

Segundo Thé (2001) essa teoria surgiu da necessidade do uso de ferramentas de modelagem mais flexíveis, cujas respostas deveriam ir além de "Verdadeiro ou Falso", "Preto ou Branco", pois muitas vezes a realidade assume diferentes graus de "Cinza". Semelhante aos problemas encontrados na modelagem da previsão climática, que entre as categorias seca e chuvosa existe um conjunto de subcategorias que deve ser levado em conta, ou seja, a realidade dos fenômenos meteorológicos em muitos casos está fora dos extremos.

A aplicação da teoria dos conjuntos difusos em estudos meteorológicos vem crescendo nos últimos anos por todo o mundo. Assim podem-se citar os estudos de Shao (2000), que fez uso da técnica para categorizar condições de tempo, mapeando características térmicas da atmosfera, de Gautan et al. (2002), que usaram a lógica Fuzzy em um modelo de estimativa de radiação solar, de Hicks et al. (2003) que apresentaram um sistema com base em lógica Fuzzy para automatizar previsões de tempo de curto prazo para fins aeronáuticos, e de Mackey (2004) que mostrou uma aplicação não linear de conjuntos difusos para combinar previsões de precipitação.

No Brasil, e em particular na região semi-árida do Nordeste do Brasil (RSANEB), em estudos de modelagem numérica e previsão climática é mais comum o uso de modelos dinâmicos (físico-matemática), e estatístico-estocásticos. Porém, Galvão (1999) mostrou uma aplicação de como essa técnica pode ser usada com objetivo de agregar informações subjetivas de especialistas, com base na configuração de variáveis atmosféricas e oceânicas, que influenciam o clima nessa região.

Nesse contexto, o objetivo desse trabalho é aplicar a teoria dos conjuntos difusos em um estudo com o objetivo de unificar informações climáticas de consenso subjetivo que são usadas na previsão climática da RSANEB, visando à obtenção da previsão determinística de um Índice Regional de Chuvas da Quadra Chuvosa (IRCQC), período de fevereiro a maio de cada ano (Alves e Repelli, 1992). Esta técnica permite um resultado determinístico agregando todas as informações climáticas usadas na previsão sazonal de chuva da RSANEB, possibilitando seu prognóstico em mais de uma categoria (MS, $\mathrm{S}, \mathrm{N}, \mathrm{C}$ e MC), informando a mais provável a vir a ocorrer em função do seu nível de pertinência.

\section{METODOLOGIA E DADOS}

Um conjunto difuso é definido por seus elementos e respectivos coeficientes de pertinência (Sugeno e Kang, 1988, Pedrycz, 1995; Lee, 1990a e 1990b). Segundo Galvão (1999), o conjunto é formalmente definido como: $\tilde{\mathrm{A}}=\left\{\left(\mathrm{x}, \mu_{\mathrm{A}(\mathrm{x})} / \mathrm{x} \in \mathrm{X}\right)\right\}$, onde $\mathrm{X}$ é o universo onde os elementos $\mathrm{x}$ estão definidos, e $\mu_{\mathrm{A}(\mathrm{x})}$ é a função de pertinência de x em Ã.

Os elementos " $\mathrm{x}$ " que pertencem a uma classe possuem graus (coeficientes) de pertinência $\left(\mu_{\mathrm{A}(\mathrm{x})}\right)$ no intervalo $[0, \mathrm{n}]$. No entanto, o intervalo de variação dos valores de $\mu_{\mathrm{A}(\mathrm{x})}$ mais usualmente empregado é $[0,1]$. Nesta situação, o conjunto difuso é dito normalizado.

Ressalta-se que as funções de pertinência dos conjuntos difusos podem assumir diversas formas. Usualmente, são selecionadas funções triangulares, trapezoidais ou Gaussianas. De todas, as triangulares são as mais comumente usadas devido a sua facilidade de aplicação. (Vieira et al., 1999).

Mais detalhes sobre a teoria dos conjuntos difusos pode ser encontrado em Sehresta e Duckstein (1989); Mandani e Assilian (1975), Zadeh (1978), Duckstein e Bogardi (1991), Kaufmman e Gupta (1991), Kosko (1992,1994); Cox (1994), Bardossy e Duckstein (1995); Sugeno (1995) e Zimmermann (1996).

A Figura 1 mostra um diagrama representativo da estrutura básica de um modelo baseado na teoria dos conjuntos difusos. Suas características são comentadas em Mamdani e Assilian (1975), citado por Paiva (1999). Em adição, tem-se uma descrição básica de cada um dos módulos contidos no modelo difuso. Pode-se observar, na Figura 1, que um modelo difuso é composto por quatro módulos fundamentais:

Módulo de Entrada (fuzificação) - Nesse módulo ocorre o processo de representação das entradas numéricas de dados através de conjuntos difusos. Assim, as incertezas inerentes a um determinado valor de uma variável numérica $x$ * são traduzidas por um conjunto difuso $\widetilde{\mathrm{X}}^{*}$, definidos num dado universo. Esta operação é fundamental, devido ao fato que a manipulação dos valores das variáveis no modelo difuso é baseada na teoria dos conjuntos difusos. Aqui são escolhidas as funções de pertinência a serem usadas (forma - triangular ou outras).

Base de conhecimento - Na construção de um modelo difuso, a seleção das regras de decisão, ou de inferência, se baseia no conhecimento prévio, conjunto de regras estabelecidas com base na intuição e/ou na experiência, de um ou mais peritos no sistema a modelar. Trata-se, de um modelo mental, também designado por algoritmo difuso. No entanto, para o desenvolvimento desses modelos, é de fundamental importância o conhecimento dos conceitos de conjunto e lógica difusa.

A base de conhecimentos subdivide-se em dois outros módulos: 
i. A base de dados que tem como função armazenar e fornecer informações do domínio físico das variáveis do sistema, necessárias ao funcionamento adequado dos módulos de fuzificação, do conjunto de regras e da desfuzificação.

ii. O conjunto de regras é um conjunto de critérios que tem como função designar as entradas e saídas do sistema difuso em termos de composições de proposições. Na construção do mesmo está envolvido a escolha das variáveis de entrada e saída do modelo, a seleção do formato dos critérios condicionais, a seleção dos termos associados a cada uma das variáveis e a síntese do conjunto de regras.

Motor de inferência - A função do motor de inferência é determinar o valor da variável do conjunto difuso de saída, com base nos parâmetros estabelecidos nos módulos de fuzificação.

Módulo de desfuzificação - A desfuzificação tem como principal objetivo a obtenção de um valor real para a saída, assim nesse módulo tem-se a aplicação de uma ou mais metodologias de transformação de conjuntos difusos, obtido na saída do modelo, em valores numéricos determinísticos (Driankov et al., 1993; Harris et al., 1993, Lee,1990a). Segundo Thé (2001), em Lee (1990b) são citados alguns trabalhos de análise destas estratégias de desfuzificação que mostram que o método do centro de gravidade é o método que permite obter melhores resultados, do ponto de vista de representar o raciocínio humano.

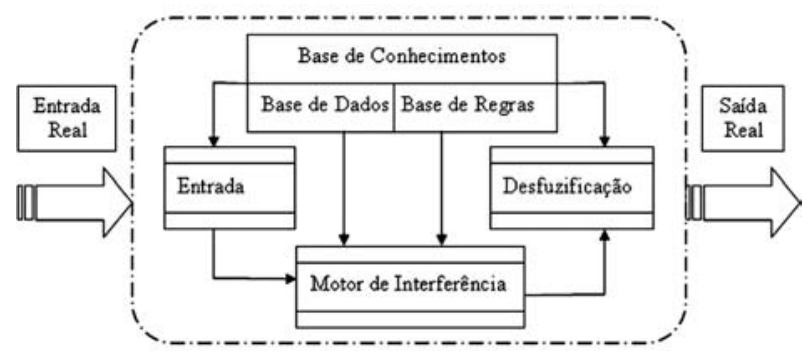

Figura 1 - Diagrama representativo de um modelo baseado na teoria dos conjuntos difusos. Figura adaptada de Paiva (1999).

No estudo em questão, as variáveis difusas de entrada se referem às características de variáveis atmosféricas e oceânicas (Tabela 1) observadas em janeiro do ano de previsão em questão, sobre e nos oceanos Atlântico (ATN) e Pacífico (PAC) Tropicais. A característica dessas variáveis atmosféricas e oceânicas tem significativa relação com as chuvas da RSANEB (Hastenrath e Heller, 1977; Moura e Shukla, 1981, Ward e Folland, 1991; Hastenrath e Greischar, 1993; Nobre e Shukla, 1996, Alves, 2002).

Vale ressaltar que a Anomalia de Temperatura da Superfície do Mar (ANTSM), é avaliada em regiões de influência no IRCQC da RSANEB, no Oceano Atlântico na região conhecida como a região do Dipolo do Atlântico, e no Oceano Pacifico na região chamada de Niño 3 (Moura e Shukla, 1981; Servain, 1991; Alves e Repelli, 1992; Alves, 2002). Essas regiões são mostradas na Figura 2.

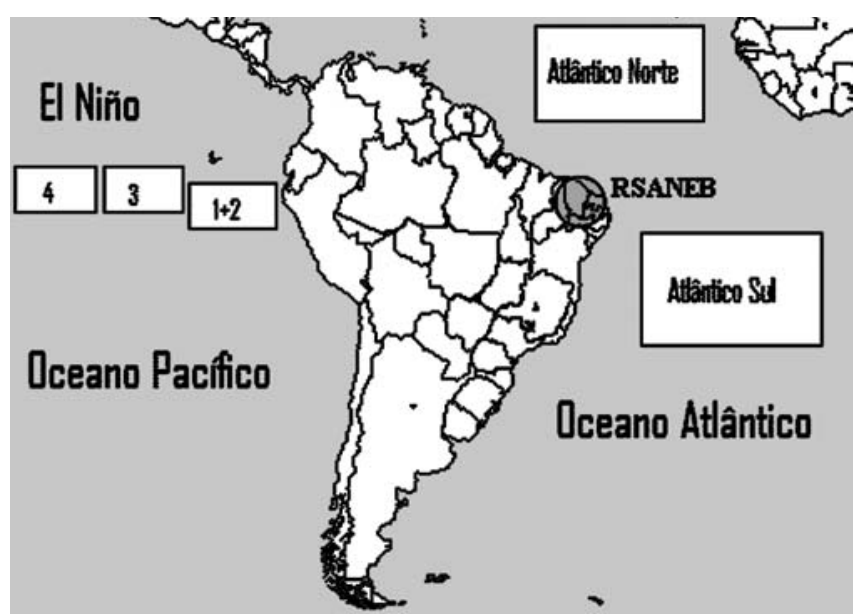

Figura 2 - Regiões onde são avaliadas as variáveis atmosféricas e oceânicas sobre e nos Oceanos Atlântico e Pacifico Tropical, e a região representativa da RSANEB no Nordeste do Brasil onde foi calculado o IRCQC.

A precipitação da RSANEB é diretamente controlada pela variabilidade da atividade convectiva, ligada diretamente as anomalias de TSM, ventos junto à superfície, pressão ao nível do mar (PNM), sobre os Oceanos Atlântico e Pacífico Tropicais (Moura e Shukla, 1981; Philander, 1990), nos parágrafos seguintes faz-se uma abordagem de como essas variáveis estão interligadas e como suas configurações (explicadas na Tabela 2), avaliadas na Tabela 1, definem características atmosféricas e condições ditas favoráveis e desfavoráveis a uma melhor distribuição de chuva na RSANEB.

A convecção tropical é dirigida pela evaporação da superfície quente dos oceanos, de modo que as variações na Temperatura da Superfície do Mar (TSM) e o seu acoplamento com a atmosfera são fatores chaves para compreender o clima global (Alves, 2003). A intensa atividade convectiva prevalece sobre as águas quentes do Oceano Pacífico e Atlântico tropicais. O desenvolvimento dessa convecção dirige as circulações de Walker e de Hadley, de modo que as variações na TSM tropical estão acopladas aos principais padrões da circulação que caracterizam o clima em escala hemisférica.

A Célula de Walker é o resultado de uma gangorra de pressão à superfície entre os setores oeste e leste ao longo do cinturão equatorial da bacia do Oceano Pacífico Tropical (Walker, 1924, 1928; Philander, 1990). Devido a essa gangorra, forma-se uma circulação zonal com movimento ascendente no setor oeste da bacia do e movimento descendente no setor leste desta (Bjerknes, 1969). Esta flutuação de pressão à superfície é denominada de Oscilação do Sul (OS). A OS tem duas fases, a positiva com as características mencionadas acima, favoráveis à chuva na RSANEB, e a fase negativa quando as características dessa gangorra entre os setores oeste e leste da bacia são contrárias. Quando ocorre a fase fria da OS, em geral, a mesma 
é relacionada a episódios El Niño, denominando-se a esse fenômeno conjunto de El Niño-Oscilação do Sul (ENOS), anomalias positivas de TSM são observadas na região de Niño 3, condição desfavorável à chuva na RSANEB. No Oceano Atlântico ocorre uma Célula de Walker secundária, associada ao chamado El Niño do Atlântico, também denominado de modo equatorial (Servain, 1998; Wang, 2002b). Neste, a circulação associada à Célula de Walker, ao longo do equador, é similar àquela observada em anos de episódios ENOS (Wang, 2002a).

A Célula de Hadley resulta da diferença de aquecimento entre os trópicos e as latitudes médias, ocasionando a formação de uma circulação meridional com movimento ascendente de ar sobre as áreas equatoriais e proximidades, e movimento descendente sobre as latitudes subtropicais de ambos os hemisférios, em torno de $30^{\circ}$ (Hastenrath, 1990; Souza e Ambrizzi, 2002). A Célula de Hadley no Oceano Atlântico está associada diretamente ao modo meridional de TSM chamado de dipólo de TSM (Servain et al., 1998). Esse modo caracteriza-se por um forte gradiente de TSM entre o Oceano Atlântico Tropical Norte (ATN) e o Oceano Atlântico Tropical Sul (ATS).

Associado a esse gradiente meridional de TSM, tem-se a formação de uma célula meridional atmosférica com duas configurações. 1) com o ar ascendente sobre a região da anomalia positiva de TSM, anomalia negativa de PNM e ventos alísios menos intensos que a climatologia, e descendente sobre a região com anomalia de TSM negativa, anomalias positivas de PNMs e área com ventos alísios mais intensos que a climatolo- gia (Wang, 2002a). Quando essas características são contrárias ocorre o segundo tipo de circulação atmosférica. No caso da circulação tipo 1 ter sido observada no setor norte da bacia do Atlântico Tropical em janeiro a previsão convencionou-se como uma condição favorável da variável analisada na Tabela 1, o contrário foi definido como condição desfavorável. Quando a configuração das variáveis mencionadas acima não apresenta características definidas em ambos os tipos de circulação, a condição foi classificada como Neutra.

Grande parte da variabilidade da chuva em FevereiroMarço-Abril-Maio (FMAM) no RSANEB é controlada pelas variações de intensidade e localização dos ramos ascendentes e descendentes das Células de Walker e de Hadley nos Oceanos Pacífico e Atlântico tropicais (Hastenrath e Heller, 1977; Moura e Shukla, 1981; Ropelewski e Halpert, 1987; Marengo, 1992; Nobre e Shukla, 1996; Uvo, 1998).

A Tabela 1 mostra dados obtidos a partir de informações de diversas variáveis atmosféricas e oceânicas cujas características foram associadas às convenções $(-),(\mathrm{N})$ e $(+)$, significando que a variável em questão tem uma condição Desfavorável (D), Neutra (N) ou Favorável (F), no sentido de inibir, não ter interferência, ou favorecer a ocorrência de chuvas na RSANEB. Essa relação observacional têm sido investigada e documentada em vários estudos climáticos e hidrológicos (Alves e Repelli, 1992; Xavier et al.; 1998).

As informações das condições das variáveis sobre e nos Oceanos Tropicais observadas em janeiro de cada ano, contidas

Tabela 1 - Condição das variáveis atmosféricas e oceânicas sobre e nos Oceanos Atlântico e Pacifico Tropical, em janeiro de cada ano, usadas como preditoras da estação chuvosa (fevereiro a maio) da RSANEB. As siglas significam: ANTSM (anomalia de Temperatura da Superfície do Mar), Setor N e Setor S (áreas localizadas no setor Norte e Sul do Atlântico Tropical), ANPNM (anomalia de Pressão ao Nível Médio do Mar), IOS (Índice de Oscilação do Sul), ANROL (anomalia de Radiação de Onda Longa), Prev. ENOS (previsão dos eventos El Niño - Oscilação do Sul), SI significa que não houve informação daquela variável no respectivo ano. Fonte: Alves et al., (2000).

\begin{tabular}{|c|c|c|c|c|c|c|c|c|c|c|c|c|}
\hline \multicolumn{13}{|c|}{ Parâmetros Atlântico } \\
\hline & 1985 & 1986 & 1987 & 1988 & 1989 & 1990 & 1991 & 1992 & 1993 & 1994 & 1995 & 1996 \\
\hline ANTSM-Setor N & + & + & + & + & + & - & $\mathrm{N}$ & $\mathrm{N}$ & $\mathrm{N}$ & + & $\mathrm{N}$ & $\mathrm{N}$ \\
\hline ANTSM-Setor S & + & + & + & + & + & - & $\mathrm{N}$ & $\mathrm{N}$ & $\mathrm{N}$ & + & $\mathrm{N}$ & $\mathrm{N}$ \\
\hline Vento- Setor N & + & + & + & + & + & $\mathrm{N}$ & $\mathrm{N}$ & - & $\mathrm{N}$ & + & $\mathrm{N}$ & $\mathrm{N}$ \\
\hline Vento- Setor S & + & + & $\mathrm{N}$ & + & + & - & $\mathrm{N}$ & $\mathrm{N}$ & $\mathrm{N}$ & $\mathrm{N}$ & $\mathrm{N}$ & $\mathrm{N}$ \\
\hline ANPNM-Setor N & + & + & $\mathrm{N}$ & $\mathrm{N}$ & + & - & $\mathrm{N}$ & - & $\mathrm{N}$ & + & + & $\mathrm{N}$ \\
\hline ANPNM-Setor S & + & + & $\mathrm{N}$ & $\mathrm{N}$ & $\mathrm{N}$ & $\mathrm{N}$ & $\mathrm{N}$ & - & $\mathrm{N}$ & $\mathrm{N}$ & $\mathrm{N}$ & $\mathrm{N}$ \\
\hline \multicolumn{13}{|c|}{ Parâmetros Pacífico } \\
\hline & 1985 & 1986 & 1987 & 1988 & 1989 & 1990 & 1991 & 1992 & 1993 & 1994 & 1995 & 1996 \\
\hline Teleconexões & + & + & + & + & $\mathrm{N}$ & $\mathrm{N}$ & + & $\mathrm{N}$ & $\mathrm{N}$ & $\mathrm{N}$ & - & $\mathrm{N}$ \\
\hline ANTSM & + & + & - & $\mathrm{N}$ & $\mathrm{N}$ & $\mathrm{N}$ & $\mathrm{N}$ & - & - & $\mathrm{N}$ & - & + \\
\hline Ventos & + & + & - & - & $\mathrm{N}$ & $\mathrm{N}$ & $\mathrm{N}$ & - & - & - & - & + \\
\hline IOS & + & + & - & $\mathrm{N}$ & $\mathrm{N}$ & $\mathrm{N}$ & $\mathrm{N}$ & - & - & $\mathrm{N}$ & SI & $\mathrm{N}$ \\
\hline ANROL & + & + & - & $\mathrm{N}$ & $\mathrm{N}$ & $\mathrm{N}$ & $\mathrm{N}$ & - & - & + & - & $\mathrm{N}$ \\
\hline Prev. ENOS & SI & SI & - & $\mathrm{N}$ & $\mathrm{N}$ & $\mathrm{N}$ & $\mathrm{N}$ & - & - & $\mathrm{N}$ & - & $\mathrm{N}$ \\
\hline
\end{tabular}


na Tabela 1, serviram de base para a definição das funções de pertinência das variáveis difusas de entrada mostradas na Figura 4. Estas são definidas pelo somatório dos eventos Desfavoráveis (D), Neutros (N) e Favoráveis (F), obtidos a partir dos dados da Tabela 1, e mostrados na Figura 3. A seguir e mostrado sucintamente como as configurações das variáveis de entrada da Tabela 1 estão associadas às convenções $\mathrm{D}, \mathrm{N}$ e F. Tais associações são atribuídas pelo conhecimento de especialistas que conhecem as configurações de tais variáveis com variabilidade climática das chuvas na RSANEB.

\section{Número de eventos $(D, N, F)$}

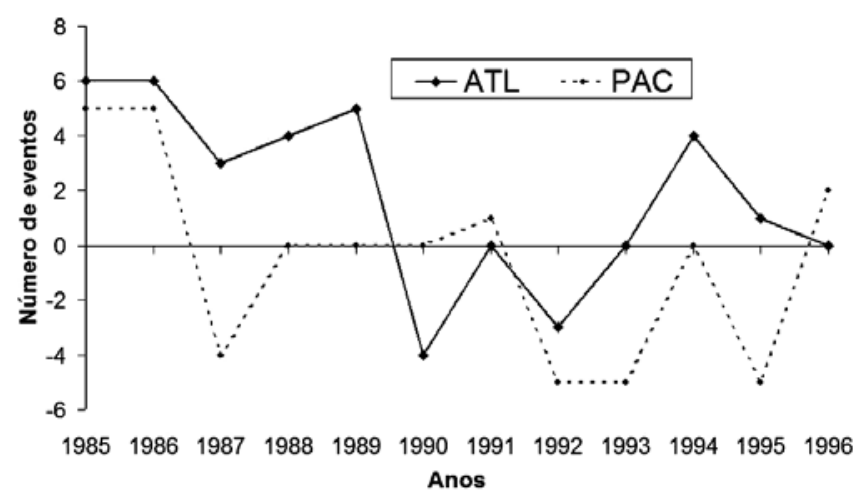

Figura 3 - Somatória dos números de eventos Desfavoráveis(D), Neutros(N) e Favoráveis (F), obtidos a partir dos dados mostrados na Tabela 1, para o mês de janeiro nos Oceanos Atlântico (ATL) e Pacifico (PAC) Tropicais.

Para as variáveis no Atlântico Tropical as seguintes configurações estão associadas as condições D, N, F:

\section{- ATSM-Setor Norte}

Favorável - Predomínio de anomalias positivas de TSM com magnitudes acima de $0,5^{\circ} \mathrm{C}$.

Desfavorável - Predomínio de Anomalias negativas de TSM com anomalias abaixo de $-0,5^{\circ} \mathrm{C}$.

Neutro - Predomínio de anomalias de TSM com valores em torno de $0,5^{\circ} \mathrm{C}$.

\section{- ATSM-Setor Sul}

Favorável - Predomínio de anomalias positivas de TSM com magnitudes acima de $0,5^{\circ} \mathrm{C}$.

Desfavorável - Predomínio de anomalias negativas de TSM com anomalias abaixo de $-0,5^{\circ} \mathrm{C}$.

Neutro - Predomínio de anomalias de TSM com valores em torno de $0,5^{\circ} \mathrm{C}$.

- APNM-Setor Norte

Favorável - Predomínio de anomalias positivas de PNM com magnitudes acima de $0,5 \mathrm{hPa}$.

Desfavorável - Predomínio de anomalias negativas de PNM com anomalias abaixo de $-0,5 \mathrm{hPa}$.
Neutro - Predomínio de anomalias de PNM com valores em torno de $0,5 \mathrm{hPa}$

- APNM-Setor Sul

Favorável - Predomínio de anomalias negativas de PNM com magnitudes abaixo de $0,5 \mathrm{hPa}$.

Desfavorável - Predomínio de anomalias positivas de PNM com anomalias acima de $0,5 \mathrm{hPa}$.

Neutro - Predomínio de anomalias de PNM com valores em torno de $0,5 \mathrm{hPa}$

- Ventos - Setor Norte

Favorável - Predomínio de anomalias positivas de ventos em $850 \mathrm{hPa}$, valores $>0,5 \mathrm{~m} / \mathrm{s}$.

Desfavorável - Predomínio de anomalias negativas de ventos em $850 \mathrm{hPa}$, valores $<-0,5 \mathrm{~m} / \mathrm{s}$.

Neutro - Predomínio de anomalias de vento em $850 \mathrm{hPa}$ com valores em torno de $0,5 \mathrm{~m} / \mathrm{s}$.

- Ventos - Setor sul

Favorável - Predomínio de anomalias negativas de ventos em $850 \mathrm{hPa}$, valores $<-0,5 \mathrm{~m} / \mathrm{s}$.

Desfavorável - Predomínio de anomalias positivas de ventos em $850 \mathrm{hPa}$, valores $>0,5 \mathrm{~m} / \mathrm{s}$.

Neutro - Predomínio de anomalias de vento em $850 \mathrm{hPa}$ com valores em torno de $0,5 \mathrm{~m} / \mathrm{s}$.

Para as variáveis no Pacífico Tropical, as seguintes configurações estão associadas as condições D, N, F:

- ATSM - Setor Equatorial da Bacia

Favorável - Predomínio de anomalias positivas de TSM com magnitudes acima de $0,5^{\circ} \mathrm{C}$.

Desfavorável - Predomínio de Anomalias negativas de TSM com anomalias abaixo de $-0,5^{\circ} \mathrm{C}$.

Neutro - Predomínio de anomalias de TSM com valores em torno de $0,5^{\circ} \mathrm{C}$.

- Ventos - $850 \mathrm{hPa}$ - Setor Equatorial da Bacia

Favorável - Predomínio de anomalias positivas de ventos em $850 \mathrm{hPa}$, valores $>0,5 \mathrm{~m} / \mathrm{s}$.

Desfavorável - Predomínio de anomalias negativas de ventos em $850 \mathrm{hPa}$, valores $<-0,5 \mathrm{~m} / \mathrm{s}$.

Neutro - Predomínio de anomalias de vento em $850 \mathrm{hPa}$ com valores em torno de $0,5 \mathrm{~m} / \mathrm{s}$.

- ANROL - Setor Centro-leste da Bacia

Favorável - Predomínio de anomalias positivas de ROL com magnitudes acima de $15 \mathrm{~W} / \mathrm{m}^{2}$

Desfavorável - Predomínio de Anomalias negativas de ROL com anomalias abaixo de $-15 \mathrm{~W} / \mathrm{m}^{2}$.

Neutro - Predomínio de anomalias de ROL com valores em torno de $15 \mathrm{~W} / \mathrm{m}^{2}$.

- PREV. ENOS

Favorável - Previsão de resfriamento das TSMs no Pacífico 
Tropical (eventos de La Niña).

Desfavorável - Previsão de aquecimento das TSMs no Pacífico Tropical (eventos de El Niño).

Neutro - Previsão de TSM em torno da média (eventos Neutros).

- Teleconexões

Favorável - Predomínio da fase negativa do padrão PNA - Pacific North-American (trens de ondas de geopotemcial do Pacífico Norte.- América do Norte e Atlântico Subtropical Norte, com a presença de anomalias negativas, positivas e negativas, respectivamente nessas regiões)

Desfavorável - Predomínio da fase positiva do padrão PNA - Pacific North-American (trens de ondas de geopotemcial do Pacífico Norte - América do Norte e Atlântico Subtropical Norte, com a presença de anomalias positivas, negativas e positivas, respectivamente nessas regiões)

Neutro - Não identificação de nenhuma das fases do padrão PNA.

Variáveis de entrada - Oceanos Atlântico e Pacifico Tropicais

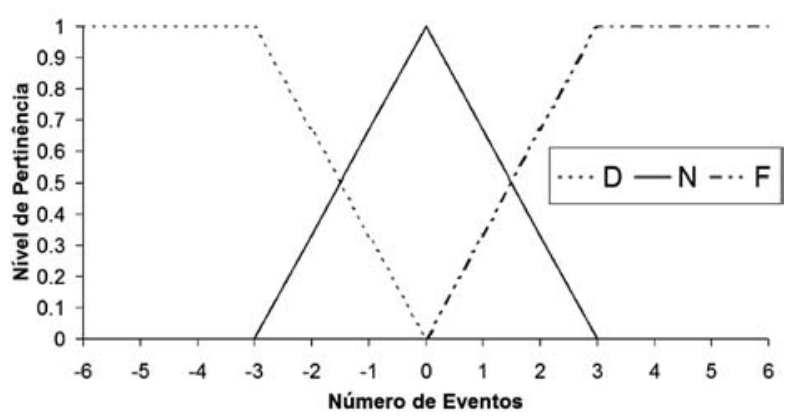

Figura 4 - Funções de pertinência das variáveis de entrada, nos Oceanos Atlântico e Pacifico Tropicais, definidas a partir dos dados contidos na Tabela 1, em três condições: D (desfavorável), N (Neutra) e F (Favorável).

A definição das regras de decisão (inferência) da variável de saída, que por sua vez é associada aos dados das variáveis de entrada é mostrada na Tabela 2. Quanto às regras de inferência, mostradas na Tabela 2 , vale mencionar que as mesmas são resultados das analiases dos especialistas com relação às configurações das variáveis da Tabela 1, e como essas estão relacionadas à condição $\mathrm{F}, \mathrm{D}$ ou $\mathrm{N}$ da variável difusa de saída (IRCQC previsto). A inferência final do prognóstico de IRCQC para cada ano (de 1985-1996) teve como base o cálculo dos valores da função de pertinência $(\mu)$ associado ao somatório dos valores de cada variável difusa de entrada, segundo critério “min-max” estabelecido por Zadeh $(1965,1978)$. Esse critério mostrado para os anos de 1991 e 1995, é definido pela análise da Tabela 1. Por exemplo, para 1991 (Tabela 3), o $\mu$ para o Pacífico
Tropical é 1,0, pois só houve informação de condições $\mathrm{N}$ para todas as variáveis analisadas, enquanto para o Atlântico Tropical houve uma divisão para condições $\mathrm{N}$ e F. Como o $\mu$ máximo é 1 para as três condições ( $\mathrm{D}, \mathrm{N}$ e F), nesse caso, para condições $\mathrm{N}$ e $\mathrm{F}$ os seus valores são dados pela proporção do somatório das análises das variáveis, o que se traduz em uma informação similar a um tercil, isto é 0,67 (para N) e 0,33 (para F). Para 1995 (Tabela 3) uma analogia similar de interpretação a Tabela 2 deve ser usada.

Tabela 2 - Regras de inferência associadas às condições das variáveis atmosféricas e oceânicas de entrada observadas sobre e nos Oceanos Atlântico (ATL) e Pacifico (PAC) Tropical (variáveis de entrada), mostradas na Tabela 1. As siglas indicam: MS (Muito Seco), Seco (S), Normal (N), Chuvoso (C) e Muito Chuvoso (MC).

\begin{tabular}{cccc}
\hline ATL/PAC & D & N & F \\
\hline D & MS & S & N \\
N & S & N & C \\
F & N & C & MC \\
\hline
\end{tabular}

É importante citar, que a técnica de conjuntos difusos, como aqui usada, não se constitui em um modelo de previsão. Nesse estudo, essa técnica é usada para otimizar as informações advindas das interpretações de especialistas, condensadas na Tabela 1, e permitir que após a aplicação da mesma seja possível uma informação determinística da previsão sazonal da chuva na RSANEB. Diferentemente da aplicação de métodos estatístico-estocástico de previsão não é necessário um período de treinamento e outro de validação do modelo.

Para o IRCQC observado na RSANEB foi utilizado um índice regional, que representa o valor médio na área compreendida entre $2^{\circ} \mathrm{S}-12^{\circ} \mathrm{S}$ e $45^{\circ} \mathrm{W}-37^{\circ} \mathrm{W}$, mostrado na Figura 5. Esse índice para o período de fevereiro a maio foi calculado pela soma dos totais pluviométricos de 66 postos com observação em cada mês de cada ano (1985-1996), e posteriormente calculada uma média. O período de anos de observação desses postos foi concomitante em todo o período de 1985-1996. A fonte desses dados é o banco de dados da antiga Superintendência de Desenvolvimento do Nordeste (SUDENE) e da Fundação Cearense de Meteorologia e Recursos Hídricos (FUNCEME). Maiores detalhes sobre esses dados são encontrados em Alves e Repelli, 1992. O período de fevereiro a maio foi escolhido porque contempla mais de $80 \%$ do total anual de chuva dessa região (Alves e Repelli, 1992). Vale ressaltar que o IRCQC observado serviu de base para a definição da função de pertinência triangular da variável de saída (IRCQC previsto), citada acima e mostrado na Figura 6. 


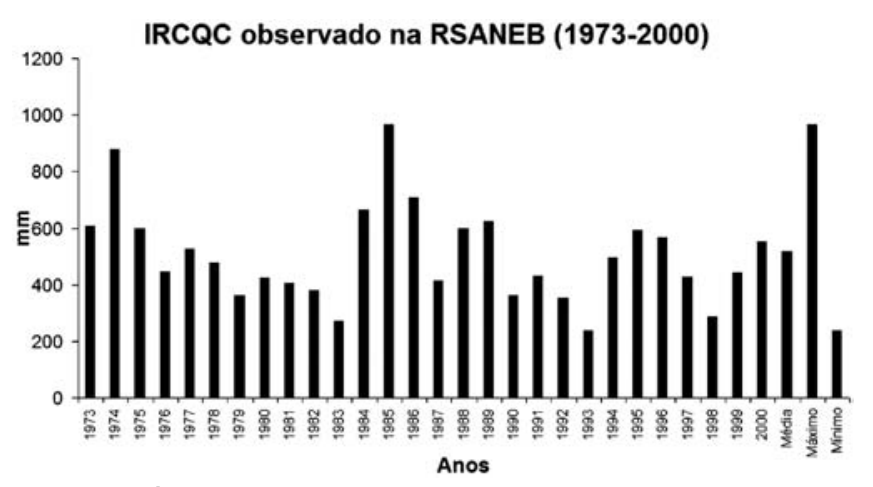

Figura 5 - Índice Regional de Chuvas na Quadra Chuvosa (IRCQC), na Região Semi-árida do Nordeste do Brasil (RSANEB), no período de 1973 a 2000, com valor máximo, mínimo e médio.

A variável difusa de saída do sistema será o IRCQC previsto na RSANEB, que será expresso em categoria definida como Muito Seco (MS), Seco (S), Normal(N), Chuvoso (C) e Muito Chuvoso (MC), associado às funções de pertinência da mesma dada pelos resultados para cada ano (1985 a 1996), cujos exemplos são mostrados na Tabelas 3 .

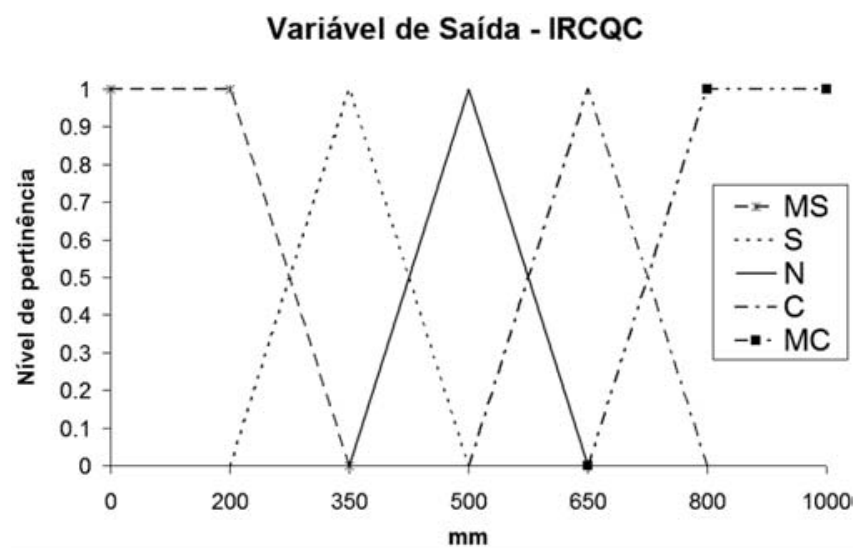

Figura 6 - Função de pertinência da variável de saída (IRCQC previsto), para a RSANEB, definido em cinco categorias: MS (Muito Seco), $\mathrm{S}$ (Seco), Normal (N), Chuvoso (C) e Muito Chuvoso (MC).

Na desfuzificação da variável difusa IRCQC previsto, que tem como objetivo obter um valor determinístico para esse índice foram usados os seguintes métodos:

1. Método do centro de gravidade ou centróide - Segundo Sugeno (1985) e Lee (1990a) este é o método fisicamente mais consistente entre os métodos de desfuzificação. Sua formulação é descrita abaixo:

$$
\mathrm{z}^{*}=\frac{\int \mu(\mathrm{z}) \mathrm{zdz}}{\int \mu(\mathrm{z}) \mathrm{dz}}
$$

2. Método dos pesos médios - Esse método considera a soma do valor médio da variável difusa de saída, em cada categoria, multiplicado pelo respectivo nível de pertinência, e dividido pela soma dos níveis de pertinência. A formulação é mostrada abaixo:

$$
\mathrm{z}^{*}=\frac{\sum \mu(\overline{\mathrm{z}}) \overline{\mathrm{z}}}{\sum \mu(\overline{\mathrm{z}})}
$$

3. Método das máximas alturas médias ou método das alturas máximas - Nesse método, calcula-se o valor médio entre os valores de máxima pertinência encontrados na variável difusa de saída. Sua formulação é:

$$
\mathrm{z}^{*}=\frac{\mathrm{a}+\mathrm{b}}{2}
$$

Onde $\mathrm{z} *$ representa a variável desfuzificada, $\mathrm{z}$ representa a variável difusa de saída (que na realidade é um limite - esse valor pode ser visto na figura 7 para o ano de 1991, variando de $300 \mathrm{~mm}$ a $800 \mathrm{~mm}$, após a sua previsão usando-se os valores das funções de pertinência encontradas, e sua plotagem em um gráfico para as categorias de IRCQC), $\mu(\mathrm{z})$ representa o nível de pertinência da variável difusa de saída, $a$ e $b$ representam os limites do intervalo de máxima pertinência encontrado da variável difusa de saída.

\section{RESULTADOS E DISCUSSÃO}

A Tabela 3 mostra um sumário da regra de inferência para os anos de 1989, 1991, 1993 e 1995. Essa tabela serviu de base para elaboração das Figuras 7 e 8 . Nota-se que para o ano de 1991 (Tabela 3) os resultados associados as variáveis de entrada da Tabela 1, após a aplicação das regras de inferência, apresentaram um nível de pertinência de $\mu(1,0)$ para a condição de $\mathrm{N}$ para as variáveis analisadas no/sobre o Oceano Pacífico. Para as variáveis analisadas no/sobre o Atlântico Tropical, as inferências mostraram um $\mu(0,67)$ para a condição $\mathrm{N}$ e $\mu(0,33)$ para a condição F. Como essas informações servem de base para a matriz de decisão a previsão para esse ano com base na definição dessa matriz foi: $\mathrm{N} \operatorname{com} \mathrm{N} \rightarrow \mathrm{N}$ e $\mathrm{N}$ com $\mathrm{F} \rightarrow \mathrm{C}$

Para 1995 (Tabela 3) os resultados das regras de inferências mostraram um nível de pertinência de $\mu(1,0)$ para a condição D para as variáveis analisadas no e sobre o Oceano Pacífico Tropical. Para as variáveis analisadas no sobre o Atlântico Tropical, as inferências mostraram um $\mu(0,67)$ para a condição $\mathrm{N}$ e $\mu(0,33)$ para a condição D. Ao cruzar com as definições atribuídas a matriz de decisão a informação do prognóstico para esse ano foi: $\mathrm{D}$ com $\mathrm{N} \rightarrow \mathrm{N}$ e D com $\mathrm{D} \rightarrow \mathrm{S}$.

Uma análise destas regras de inferências foi feita para anos de contrastes climáticos (1989-chuvoso e 1993-seco). No ano de 1989 (Tabela 3) dessas regras mostraram um nível de pertinência de $\mu(1,0)$ para a condição D para as variáveis analisadas no e sobre o Oceano Pacífico Tropical. Para as variáveis 
analisadas no sobre o Atlântico Tropical, as inferências mostraram um $\mu(0,67)$ para a condição $C$ e $\mu(0,33)$ para a condição $\mathrm{N}$. Como resposta ao cruzamento dessas informações vindas da matriz de decisão a previsão para o referido ano foi: $\mathrm{D}$ com $\mathrm{N}$ $\rightarrow \mathrm{N}$ e $\mathrm{D}$ com $\mathrm{D} \rightarrow \mathrm{S}$.

No caso de 1993 (Tabela 3), a síntese das regras de inferências resultou em: $\mu(1,0)$ para a condição D para as variáveis analisadas no e sobre o Oceano Pacífico Tropical. Para as variáveis analisadas no sobre o Atlântico Tropical, as inferências mostraram um $\mu(0,67)$ para a condição $\mathrm{D}$ e $\mu(0,33)$ para a condição N. Ao final do cruzamento dessas informações vindas da matriz de decisão o prognóstico para esse ano foi: D com $\mathrm{D} \rightarrow \mathrm{S}$ e $\mathrm{D} \operatorname{com} \mathrm{N} \rightarrow \mathrm{N}$.

As Figuras 7 e 8 mostram os resultados da aplicação da técnica de conjuntos difusos para a previsão do IRCQC para a RSANEB. Nessas Figuras apresenta-se um exemplo gráfico de como pode ser apresentada à previsão do IRCQC sem a desfuzificação para os anos de 1991 e 1995. Pode-se observar por essas

Tabela 3 - Resultado da aplicação da regra de inferência de acordo com os resultados do somatório das variáveis de entrada da Tabela 1 , com as respectivas categorias e níveis de pertinência $(\mu)$ para os anos de 1989, 1991, 1993 e 1995.

\begin{tabular}{|c|c|c|c|c|}
\hline ATL/PAC & $\begin{array}{l}\mu \\
\downarrow\end{array}$ & D & $\mathbf{N}$ & $\mathbf{F}$ \\
\hline \multicolumn{5}{|c|}{1989} \\
\hline$\mu \rightarrow$ & & & 1.00 & \\
\hline \multicolumn{5}{|l|}{$\mathrm{D}$} \\
\hline \multicolumn{5}{|l|}{$\mathrm{N}$} \\
\hline $\mathrm{F}$ & 1.00 & & $\mathrm{C}(1.00)$ & \\
\hline \multicolumn{5}{|c|}{1991} \\
\hline$\mu \rightarrow$ & & & 0.67 & 0.33 \\
\hline \multicolumn{5}{|l|}{$\mathrm{D}$} \\
\hline $\mathrm{N}$ & 1.00 & & $\mathrm{~N}(0.67)$ & $\mathrm{C}(0.33)$ \\
\hline \multicolumn{5}{|l|}{$\mathrm{F}$} \\
\hline \multicolumn{5}{|c|}{1993} \\
\hline$\mu \rightarrow$ & & 1.00 & & \\
\hline \multicolumn{5}{|l|}{$\mathrm{D}$} \\
\hline $\mathrm{N}$ & 1.00 & $\mathrm{~S}(1.00)$ & & \\
\hline \multicolumn{5}{|l|}{$\mathrm{F}$} \\
\hline \multicolumn{5}{|c|}{1995} \\
\hline$\mu \rightarrow$ & & 1.00 & & \\
\hline \multicolumn{5}{|l|}{ D } \\
\hline $\mathrm{N}$ & 0.67 & $\mathrm{~S}(0.67)$ & & \\
\hline $\mathrm{F}$ & 0.33 & $\mathrm{~N}(0.33)$ & & \\
\hline
\end{tabular}

figuras que essas ressaltam as categorias da variável de saída prevista em cada ano, e qual é a mais provável de vir a ocorrer segundo o seu maior nível de pertinência (Zadeh, 1978).

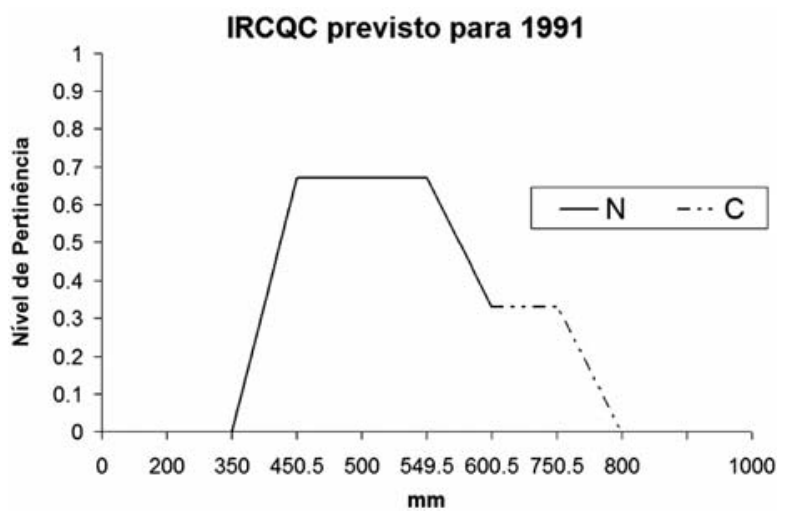

Figura 7 - Previsão obtida pela técnica dos conjuntos difusos para 1991. Método de desfuzificação 1 (método do centro de gravidade).

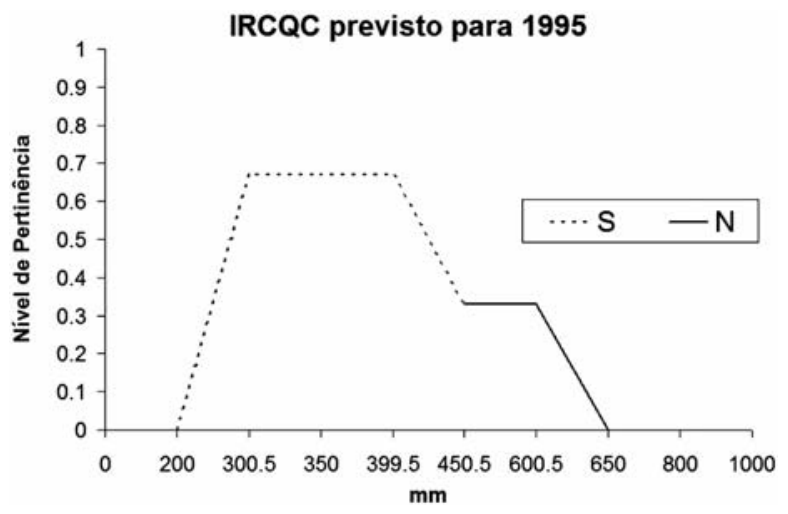

Figura 8 - Previsão obtida pela técnica dos conjuntos difusos para 1995. Método de desfuzificação 1 (método do centro de gravidade).

A Tabela 4 apresenta uma comparação entre os valores do IRCQC observado e os valores determinísticos encontrados a partir da otimização usando conjuntos difusos da previsão desse índice por categorias, e também uma comparação por categoria, para os 12 anos do estudo. Nota-se que, a previsão usando esta técnica, tanto por categorias como pela obtenção dos valores determinísticos é consistente com o observado. Em geral a previsão, usando conjuntos difusos, conseguiu capturar uma das categorias na qual o IRCQC observado se inseriu.

Em adição, calculando-se a correlação entre os valores do IRCQC observados e os valores determinísticos previstos, pelos três métodos utilizados, encontra-se que a correlação entre os valores observados e os valores obtidos pelo método do centro de gravidade (Método 1) é de 0,85, entre os valores observados e os encontrados pelo método dos pesos médios (Método 2) é de 0,81 , e entre os valores e os obtidos pelo método das alturas (Método 3) é de 0,80 . 
Tabela 4 - IRCQC observado (mm), categorizado, previsto por categorias e a previsão de valores determinísticos (valores desfuzificados - mm), pelos três métodos de desfuzificação.

\begin{tabular}{ccccccc}
\hline & Observado & & \multicolumn{5}{c}{ Previsto } \\
\cline { 6 - 7 } & Observado & Categoria & Categoria & Método 1 & Método 2 & Método 3 \\
\hline 1985 & 965 & MC & MC & 864 & 825 & 900 \\
\hline 1986 & 708 & C-MC & MC & 864 & 825 & 900 \\
\hline 1987 & 415 & S-N & N & 500 & 500 & 500 \\
1988 & 598 & N-C & C & 650 & 650 & 650 \\
\hline 1989 & 622 & N-C & C & 650 & 650 & 650 \\
\hline 1990 & 361 & S-N & S & 350 & 350 & 350 \\
\hline 1991 & 429 & S-N & N-C & 550 & 550 & 500 \\
\hline 1992 & 352 & S-N & MS & 140 & 175 & 100 \\
\hline 1993 & 237 & MS-S & S & 350 & 350 & 350 \\
\hline 1994 & 494 & N-C & C & 650 & 650 & 650 \\
\hline 1995 & 591 & N-C & S-N & 529 & 400 & 350 \\
\hline 1996 & 568 & N-C & C-MC & 550 & 550 & 500 \\
\hline
\end{tabular}

\section{CONCLUSÕES E RECOMENDAÇÕES}

Nesse estudo, é apresentada uma aplicação dos conjuntos difusos na otimização da previsão de consenso do IRCQC para a RSANEB usando como variáveis de entrada as características de variáveis atmosféricas e oceânicas observadas, em janeiro do ano de previsão, sobre e nos Oceanos Pacífico e Atlântico Tropicais para o período de 1985-1996. Os resultados mostraram que a técnica dos conjuntos difusos usada como ferramenta de otimização das variáveis supras citada, previu pelo menos uma das categorias da variável de saída (IRCQC), na qual se inseriu esse total de chuva observado nessa região, no ano da previsão.

Quantitativamente, na variabilidade interanual, os resultados foram mais próximos do observado para os anos classificados nas categorias de Normal a Muito Chuvoso, apresentando maiores diferenças para os anos Secos e Muito Secos. A comparação para os 12 anos de estudo entre o total de chuva observado e o previsto na RSANEB, embora a amostra estatística seja pequena para conclusões categóricas, mostrou correlações que variam de 0,8 a 0,85 dependendo do método de desfuzificação usado. Sendo que o método que apresentou maior correlação entre valores previstos e os observado foi o método do centro de gravidade, concordando com o sugerido por Sugeno (1985) e Lee (1990a). Esses valores de correlação, ressaltando-se que a otimização é feita com a análise de variáveis de janeiro, se constituindo em um prognóstico, são da mesma ordem dos encontrados pelos métodos estatísticosestocáticos e dinâmicos que prognosticam a chuva sazonal da RSANEB (Ward e Folland, 1991; Hastenrath e Greischar, 1993; Xavier et al., 1998 e Sun et al., 2005).
A importância da aplicação da técnica dos conjuntos difusos é que esta otimiza informações advindas de configurações variáveis oceânicas e atmosféricas que tem relação com a qualidade da quadra chuvosa da RSANEB. Além disso, a mesma permite, um resultado determinístico do IRCQC previsto, e uma a informação da sua previsão em mais de uma categoria, possibilitando uma informação de qual é mais provável de ocorrer, em função do valor do seu nível de pertinência.

Na impossibilidade de acesso, de resultados de previsão dinâmica, o uso de conjunto difuso pode auxiliar, a previsão sazonal de chuva da RSANEB fazendo uma otimização do resultado de consenso das variáveis preditoras, vindo da discussão de especialistas em climatologia dinâmica. Um das restrições do uso dessa técnica de otimização, é que quanto maior for o número de informações (variáveis de inferências - aqui nesse estudo condensamos em três, N, F e D - as informações das diversas variáveis oceânicas e atmosféricas usadas), maior será a matriz de decisão das variáveis de entrada do sistema, sendo necessário softwares especializados para gerarem as regras de inferências.

Para estudos futuros sugere-se o aprofundamento dessa metodologia, dando-se pesos diferentes às variáveis de entrada, com um maior número de categorias, aprimorando-se a matriz de decisão, e avaliando-se um conjunto maior de anos.

\section{AGRADECIMENTOS}

Os autores agradecem a Coordenação de Aperfeiçoamento de Pessoal de Nível Superior (CAPES) e a Fundação Cearense de Apoio ao Desenvolvimento Científico e Tecnológico 
(FUNCAP) pelo suporte financeiro dados para a realização desse trabalho. Os autores também são gratos aos revisores anônimos da RBMET cujas críticas e sugestões muito contribuíram para elucidar alguns pontos do artigo.

\section{REFERÊNCIAS BIBLIOGRÁFICAS}

ALVES, J. M. B., et al. Uma aplicação da técnica de "downscaling" dinâmico no setor norte da região Nordeste do Brasil. Revista Brasileira de Meteorologia, v.18, n.2, p.163-182. 2003.

ALVES, J. M. B. Reanálise de índices de temperatura da superfície do mar no Atlântico e Pacífico tropicais potenciais indicadores da qualidade da estação chuvosa do setor norte do Nordeste do Brasil. Revista Brasileira de Agrometeorologia, v.10, n.1 p.179-187. 2002.

ALVES, J. M. B., et al. Verificação de prognósticos sazoanais de precipitação no estado do Ceará utilizando a técnica dos quantis. Revista Brasileira de Meteorologia, v.15, n.2, p.73-85. 2000 .

ALVES, J. M. B., REPELLI, C. A. A variabilidade pluviométrica no setor norte do Nordeste e os eventos El Niño-Oscilação Sul (ENOS). Revista Brasileira de Meteorologia, v.7, n.2, p.583-92, 1992.

BARDOSSY, A.; DUKSTEINS, L. Fuzzy rule-based modeling in geophysical economic, and enginnering systems. Boca Raton: CRC Press, 1995.

BJERKNES, J. Atmospheric teleconnections from the equatorial Pacific. Mon. Wea. Rev., v.97, p.163-172. 1969.

COX, E. The fuzzy systems handbook. Boston: AP Professional, 1994.

DRIANKOV, D., HELLENDOORN, H. e REINFRANK, M. An Introduction to Fuzzy Control. Berlin: SpringerVerlag, 1993.

DUKSTEIN, L; BOGARDI, I. Reliability with fuzzy elements in water quantity an problems. In: GANOULIS, J. (ED). Water RESOURCEsw enginnering risk assessment. Berlin: Springer-Verlag, 1991.

GALVÃO, C. O. Introdução a Teoria dos Conjuntos Difusos. Sistemas Inteligentes. Aplicações a Recursos Hídricos e Sistemas Ambientais. Capítulo 5. Org. Galvão, C. O.; Mêuser, J. S. Porto Alegre, ABRH, p. 246. 1999.
GAUTAM, N. K., Kaushika, N. D. A Model for the Estimation of Global Solar Radiation Using Fuzzy Random Variables. J. Appl. Meteor., vol. 41, n.12, p. 1267-1276, 2002.

HARRIS, C. J., et al. Intelligent Control - Aspects of Fuzzy Logic and Neural Nets. Singapore: World Scientific Publishing, 1993.

HASTENRATH, S.; HELLER, L. dynamics of climatic hazards in northeast Brazil. Quarterly J. Roy. Meteor. Socie., v.103, n.435, p.77-92. 1977

HASTENRATH, S.; GREISCHAR, L. Circulation mechanisms related to Northeast Brazil rainfall anomalies. J. Geophys. Res., v.98, n.d, p.5093-102, 1993.

HICKS, T. An Improved Fuzzy Logic System For Automated Short Term Aviation Weather Forecasts. In: AMS Conference on Aviation, Range, and Aerospace, 11., Hyannis, MA, p.4/8, October. 2004.

KAUFMANN, A; GUPTA, M. M. Introduction to fuzzy aritthmetic-theory and applications. New York: Van Nostrand Reinhold, 1991.

KOSKO, B. Neural networks and fuzzy systems. Englewood Cliffs: Prentice-Hall, 1992.

KOSKO, B. Fuzzy thinking. London: Flamimgo, 1994.

LEE, C. C. Fuzzy logic in control systems: fuzzy logic controller - part I. IEEE Transactions on Systems, Man and Cybernetics. v. 20, n. 2, p. 404-418. 1990a.

LEE, C. C. Fuzzy logic in control systems: fuzzy logic controller - part II. IEEE Transactions on Systems, Man and Cybernetics. v. 20, n. 2, pp. 419-435. 1990b.

MACKEY, B. P. A non-linear fuzzy set technique for combining precipitation forecasts. Conference on Weather Analysis and Forecasting, 20., Conference on Numerical Weather Prediction, 16. American Meteorological Society, 11-15 January, Seattle, Washington. 2004.

MAMDANI, E. H., ASSILIAN, S. An experiment in linguistic synthesis with a fuzzy logic controller. Inter. Jour. ManMachine Stu., v.7, p.1-13, 1975.

MARENGO, J. Interannual variability of surface climate in the Amazon basin. Int. J. Clim., v. 12, p.853-863. 1992. 
MOURA, A. D.; SHUKLA, J. On the dynamics of droughts in northeast Brazil: observations, theory and numerical experiments with a general circulation model. J. Atmos. Scie., v.38, n.12, p.2653-2675. 1981.

NOBRE, P.; SHUKLA, J. Variations of sea surface temperatures, wind stress, and rainfall over the tropical over the tropical Atlantic and South America. J. Climate, v.9, n.10, p.24642479. 1996.

PAIVA, R. P. P. de C. Identificação Neuro-Difusa Aspectos de Interpretabilidade. 1999. 163p. Dissertação (Mestrado) - Faculdade de Ciências e Tecnologia, Universidade de Coimbra, Coimbra.

PEDRYCZ, W. Fuzzy Sets Engineering, CRC Press. 1995.

PHILANDER, S. G. El Niño, La Niña, and Southern Oscillation. Londres: Academic Press, 1990. 289p.

ROPELEWSKI, C. F.; HALPERT, M. Global and regional scale precipitation patterns associated with the El Niño/Southern Oscillation. Mon. Wea. Rev., v.115, p.1606-1626. 1997.

SHAO, J. Fuzzy Categorization of Weather Conditions for Thermal Mapping. J. Appl. Meteor., v. 39, n.10, p. 1784 1790. 2000.

SERVAIN, J. Simple climatic índices for the tropical Atlantic Ocean and some aplications. J. Geophy. Res., v.96, n.C8, p.15.137-15.146. 1991 .

SERVAIN, J. et al. A pilot research moored array in the tropical Atlantic (PIRATA). Bull. Amer. Meteor. Socie., v.79, n.10, p.2019-2031.1998.

SHRESTHA, B.; DUCKSTEIN, L. A fuzzy reliability measure for enginnering applications. In: Uncertainty modeling and analysis in civil engineering. Boca Raton: CRC Press, 1989.

SOUZA, E. B.; AMBRIZZI, T. ENSO impacts on the South American rainfall during 1980s: Hadley and Walker circulations. Atmos., v.15, p.105-120. 2002.

SUGENO, M. Industrial applications of fuzzy control. Boston: North-Holland, 1995.

SUGENO, M. e KANG, G. T. Structure identification of fuzzy model, Fuzzy Sets and Systems,v. 28, p. 15-33. 1988.
SUN, L.; MONCUNNIL, D. F.; LI, H.; MOURA, A. D.; FILHO, F. D. D. S. Climate downscaling over Nordeste Brazil using NCEP RSM97. J. Climate, v.18, p.551-567. 2005.

THÉ, M. A. L. Raciocínio Baseado em Casos: Uma Abordagem Fuzzy para Diagnóstico Nutricional.. 2001. 170 p. Tese de Doutorado - Programa de Pós-graduação em Engenharia de Produção, Universidade Federal de Santa Catarina,. Florianópolis-SC.

UVO, C. Influence of Sea Surface Temperature on Rainfall and Runoff in Northeastern South America: Analysis and Modelling. 1998. 120p. Phd. Diss. Department of Water Resources Engineering, Lund University, Lund, Sweden.

WALKER, G. T. Correlation in seasonal variations of weather. IX a further study of the world weather. Mem. Indian Meteorol. Dep., v.24, p.275-332. 1924.

WANG, C. Atmospheric circulation cells associated with the El Niño-Southern Oscillation. J. Climate, v.13, p.39693993. 2002a.

WANG, C. Atlantic climate variability and its associated atmospheric circulation cells. J. Climate, v.15, p.15161536. $2002 b$.

WARD, M. N.; FOLLAND, C. K. 1991. Prediction of seasonal rainfall in the north nordeste of Brazil using eingenvectors of sea-surface temperature. Int. J. Clim., v.11, 711-43. 1991.

VIEIRA, V. P. P. B. Avaliação Quantitativa de Riscos Econômicos e Ambientais. Sistemas Inteligentes. Aplicações a Recursos Hídricos e Sistemas Ambientais. Porto Alegre: Capítulo 5. Org. Galvão, C. O.; Mêuser, J. S. ABRH, p. 246. 1999.

XAVIER, T. de Ma. B. S., et al. Papel da componente meridional do vento na costa norte do Nordeste brasileiro e de outras covariáveis para prever a chuva no Estado do Ceará (196467). Rev. Bras. Rec. Hídri., v.3, n.4, p.121-39. 1998.

ZADEH, L. A. Fuzzy Sets. Information and Control, v.8. p.338-353, 1965.

ZADEH, L. Fuzzy sets as basis for theory of possibility. Fuzzy Sets and systems, v.1, p.3-28, 1978.

ZIMMERMANN, H, J. Fuzzy set theory and its applications. 3ed. B oston: Kluwer Academic Publishers, 1996. 